\title{
Artificial Finger Skin having Ridges and Distributed Tactile Sensors used for Grasp Force Control
}

\author{
Daisuke Yamada, Takashi Maeno and Yoji Yamada
}

\begin{abstract}
An artificial elastic finger skin for robot fingers has been developed for controlling grasp force when weight and frictional coefficient of the grasped object are unknown. The elastic finger skin has ridges at the surface to divide the stick/slip area. It also has a pair of tactile sensors embedded per one ridge similar to human fingertips. The surface of the whole finger is curved so that reaction force distributes. A Finite Element (FE) model of the elastic finger skin was made to conduct a dynamic contact analysis using a FE method in order to design the elastic finger skin in detail. Then the elastic finger skin was made. As a result, it was confirmed by calculation and experiment that the incipient slippage of the ridge that occurs near the edge of contact area is detected. Then, a grasp force was controlled using the finger. Arbitrary object was lifted successfully by use of incipient slippage near the edge of contact area. Consequently, it was found that the artificial finger skin is useful for controlling grasping force when the weight and friction coefficient between the elastic finger skin and grasping object are unknown.
\end{abstract}

\section{Introduction}

Epidermal ridges are located at the surface of the fingers of humans. Papillae are arranged in two lines underneath the ridge. FA I and FA II tactile receptors are also arranged in two lines underneath the ridge. Maeno et al. [1] showed by finite element (FE) analysis that this arrangement is useful for enlarging the sensitivity of the tactile receptors. It was also shown that the structure of epidermal ridges is correlated to the partial insipient slippage between the fingers and object. This structure also seems to be useful for tactile receptors to detect signals for controlling an adequate force to grip and lift an object without slippage.

On the other hand, the use of a robot hand to grip and lift an object is one of the most important problems in the field of robotics. Several solution methods have been presented. One method is to pay attention to the detection of partial incipient slippage between the finger and object. This method is thought to be similar to what humans do [2][3]. The partial incipient slippage refers to the contact condition between the robot fingers and the grasped object in which part of the contact area slips while the other part sticks. This method was proposed first by Trembley et al. [4]. They detected the partial incipient slippage of finger skin by sensing micro vibrations, which are caused by an expansion of the slip regions within the contact area when the tangential force increases. The micro vibrations are detected by two accelerometers near the skin. However, entire slippage often occurs immediately after the micro vibrations occur. This is because the location of the partial incipient slippage is not specified and so small partial incipient slippage cannot be detected when it locally occurs near the edge of the contact area. The stick/slip distribution between the finger and object should be measured to control grasping and lifting force precisely. De Rossi et al. [5] made a device covered with silicone rubber and embedded distributed PVDF films as tactile sensors underneath the silicone rubber. The distributed tactile sensors can selectively be sensitive to shear and normal stress components. A neural network, whose inputs are outputs from the tactile sensors, provides a global sliding coefficient as an output. The partial incipient slippage between the device and objects were investigated. However, accuracy for detecting the global sliding slippage is not high enough, because the location of partial incipient slippage was not specified due to the flat surface of the device. Maeno et al. [6] showed that the shear strain distribution pattern inside a curved elastic finger indicates the stick/slip distribution at the finger surface during the precision grip. The partial incipient slippage always occurs near the edge of contact area by use of the curved finger. They also proposed a sensor detecting the strain distribution pattern inside the elastic finger having a curved surface instead of using the detected value itself [7][8]. The strain distribution pattern is measured by strain gages embedded in the elastic finger. They showed a method for controlling the grasping force by use of proposed elastic robot finger as well. However, the object must be lifted at a certain speed. If the weight of object is changed while lifting the object, the object will not remain grasped. Other methods to detect slippage have been presented. Yamada et al. [9] showed a slip sensor that has elastic ridges at the surface and is capable of isolating a stick-slip vibration due to an entire slippage between the sensor and grasping object. The sensor can detect the entire slippage and control the grasping force 
quickly and correctly to avoid dropping the object. However, the method is not adequate because the position of the object changes slightly due to the entire slippage.

In this study, an artificial elastic finger skin that has a unique geometry imitating the configuration of ridges and FA I receptors of the human finger was designed and manufactured by combining the method proposed by Maeno et al. [8] and Yamada et al. [9]. Then it is shown that it can detect the incipient slippage of the ridges near the edge of the contact area.

A design of the elastic finger skin is described in section 2. A model and results of FE analyses are shown in section 3. An experiment to confirm the analysis is presented in section 4. An experiment which arbitrary object is lifted by controlling a grasping force is show in section 5. The conclusions of this study are described in section 6 .

\section{Design of the artificial finger skin}

\subsection{What to mimic in human fingers}

The artificial finger skin can be designed by imitating the characteristics of human fingers because humans can grasp an object at arbitrary finger speeds without producing entire slippage, even when the weight and coefficient of friction between the finger and the object are unknown. The characteristics we paid attention to are as follows: First, the human finger consists of flexible material and has a curved surface. Then a partial incipient slippage easily occurs near the edge of contact area between the finger and the object. Normal reaction force at the center of the contact area is larger than that near the edge of the contact area. However tangential reaction force is almost equal throughout the contact area. Therefore, the center of contact area generally "sticks". On the other hand, the edge of the contact area easily changes its contact condition from "stick" to "slip". Because of this, the partial incipient slippage occurs near the edge of the contact area. Second, epidermal ridges are distributed at the surface of the finger. When the partial incipient slippage occurs, constraints due to the "stick" condition of the ridges near the edge of the contact area are released. Then, elastic deformation of the ridges is suddenly reduced. Furthermore, speed of movement of the slipping ridge is constant depending on the natural frequency of the ridge, even when both the frictional coefficient between the finger and object and the applied force changes. Third, a pair of FA I receptors are located at the tip of papillae which are arranged in two lines underneath the epidermal ridge. A stress concentration occurs at the location of FA I receptors. A strain distribution inside the ridge is closely related to the deformation of the ridges as shown in Fig. 1. Strain at the location of the pair of FA I receptors is equal when only the normal force is applied to the ridge as shown in Fig. 1 (a). On the other hand, the strains differ from each other when both the normal and tangential forces are applied to the ridges as shown in Fig. 1 (b). Now, we can conclude that the deformation of the ridge in the tangential direction can be detected by subtracting the strains at the two points underneath the ridge. Particularly, when the partial incipient slippage occurs, a velocity of the subtracted value must decrease largely and an acceleration of the subtracted value becomes a negative impulse at that time.

It is expected that the incipient slippage of each ridge can be detected by use of the above-mentioned velocity and acceleration of strain underneath each ridge. Information about the incipient slippage can be used as inputs for controlling the grasping force, even when the weight and frictional coefficient of the object and the applied force are unknown.

\subsection{Detailed design}

The artificial finger skin is designed in detail to imitate the above-mentioned characteristics of the human finger.

First, the shape of the ridges was decided. A FE model of one ridge was made for conducting a dynamic contact analysis using a FE method. The shape of the ridge consists of a combination of several arcs. The frictional coefficient was set at 1.0 in the analysis. Material properties of the model that are similar to those of silicone rubber are as follows:

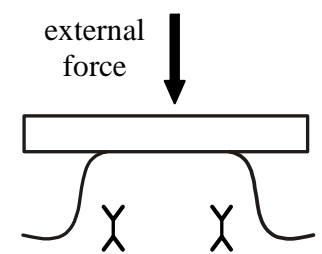

equal compressive strain

(a) Normal force is applied

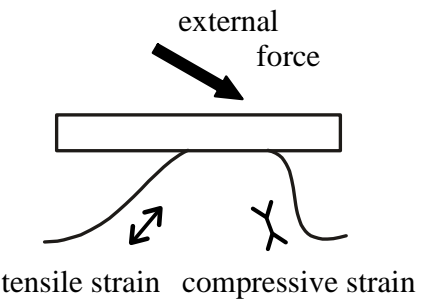

(b) Normal and tangential forces are applied

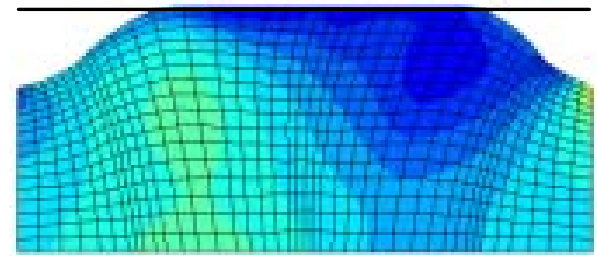

Fig. 2 Distribution of shear strain inside the ridge

Fig. 1 Distribution of strain inside the ridge 
Young's modulus of the artificial finger skin is 4.96 $\mathrm{MPa}$. Poison's ratio is 0.49 . Mass density is 1230 $\mathrm{kg} / \mathrm{m}^{3}$. Mechanical material type of finger is isotropic. A flat object, defined as a rigid body, was set to be in contact with the model from the top. Then, an external force was applied by changing the position of the flat object. Figure 2 shows the distribution of strain inside the ridge when the normal and tangential movements of the flat object were applied. The FE program MARC was used. As expected, shear strain underneath the right hand edge of the ridge is large, Fig. 1 (b). Now, we can say that the shape of the ridge is adequate.

Second, locations and directions to detect the pair of strain inside the ridge were decided. A relationship between the directions of external force and principal strain inside the ridge is analyzed. The results are as follows: When only normal force is applied, the direction of principal strain at the location corresponding to that of FA I receptors is almost parallel to the direction of external force.

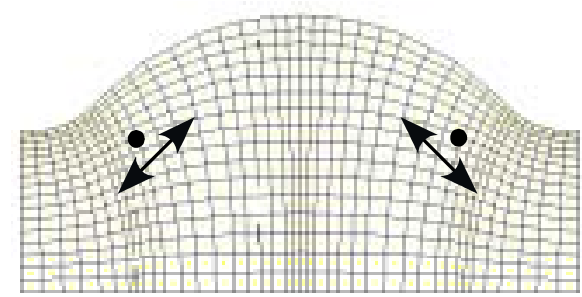

Fig. 3 Points and directions for subtracting the strains

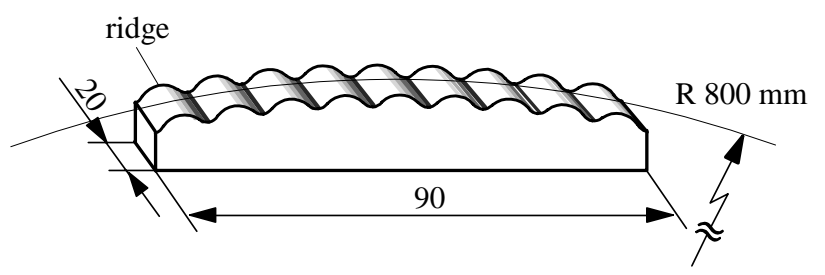

Fig. 4 Top view of designed artificial finger skin
However, when both normal and tangential forces are applied, the direction of principal strain at the same location is inclined to about 45 degrees. Consequently, a relationship between the tangential deformation of the ridge and the subtracted value of strains can be calculated. So, the location and direction were decided as shown in Fig. 3.

Third, a radius of curvature for the artificial finger skin was decided. If the radius is too large, normal contact force does not distribute and the location of the incipient slippage of the ridges cannot be specified near the edge of contact area. If the radius is too small, the contact area is too small and ridges at the edge of the artificial finger skin are not in contact, even when a large normal force is applied. The radius was decided to be $800 \mathrm{~mm}$ by considering the above-mentioned condition. Results of FE analysis show that all the ridges are in contact with a flat object when the normal force of about $4 \mathrm{~N}$ is applied, so the assumed limit of the weight of the object is about $8 \mathrm{~N}$ when the object is grasped by two fingers. The number of ridges was set at nine to detect the partial incipient slippage selectively, even when the contact area changes due to the change in value and direction of normal contact force. The designed artificial finger skin is shown in Fig. 4.

\section{Numerical analysis}

\subsection{FE model of the artificial finger skin}

A FE model of the entire artificial finger skin is made as shown in Fig. 5 for analyzing the dynamic contact condition between the finger and a flat object. A purpose of the analysis is to confirm that the entire slippage of the ridge near the edge of contact area can be detected by use of the velocity and acceleration of strains inside the ridge as predicted in section 2 , even when the applied force, the friction coefficient and the increment of applied force are changed. The position of the bottom edge of the artificial finger skin was fixed. The ridges are numbered from one to nine for identifying each other. Material properties, material type and applied external forces of the model are the same as those of the analysis described in section 2 .

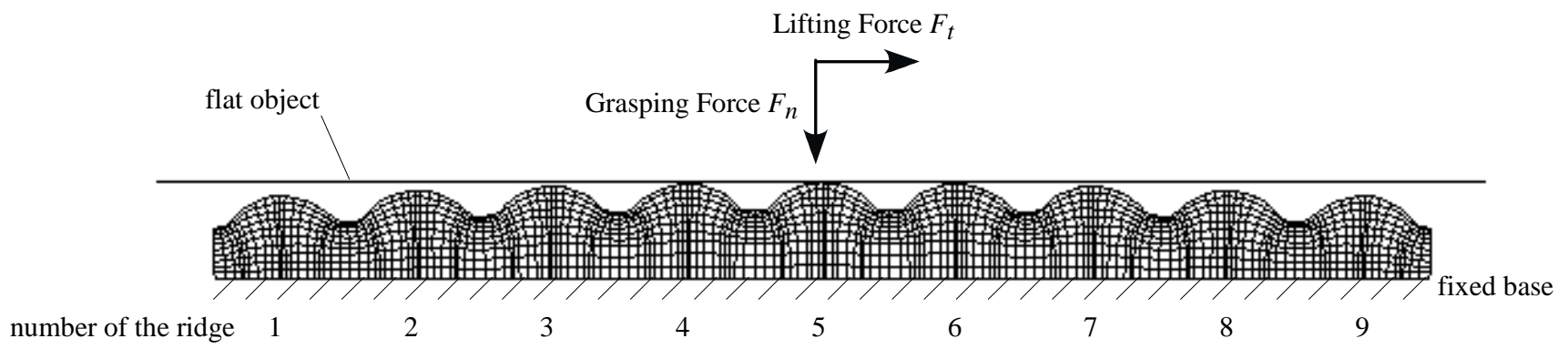

Fig. 5 Two dimensional FE model of designed artificial finger skin 


\subsection{Condition of calculation}

The external force, i.e. grasping force $F_{n}$ and lifting force $F_{t}$ (see Fig. 5), was increased as shown in Fig. 6. Only the grasping force was applied at first for avoiding entire slippage when the lifting force is initially applied same as human do. Then, the grasping and lifting forces were increased. The partial

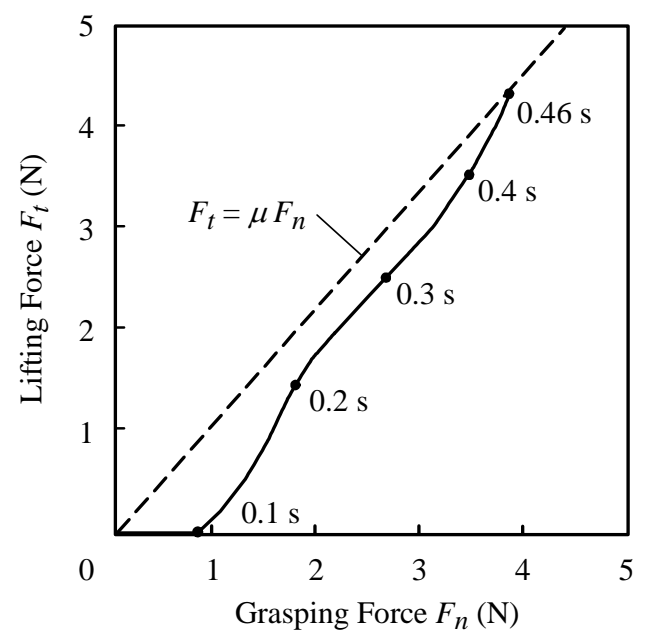

Fig. 6 History of external force

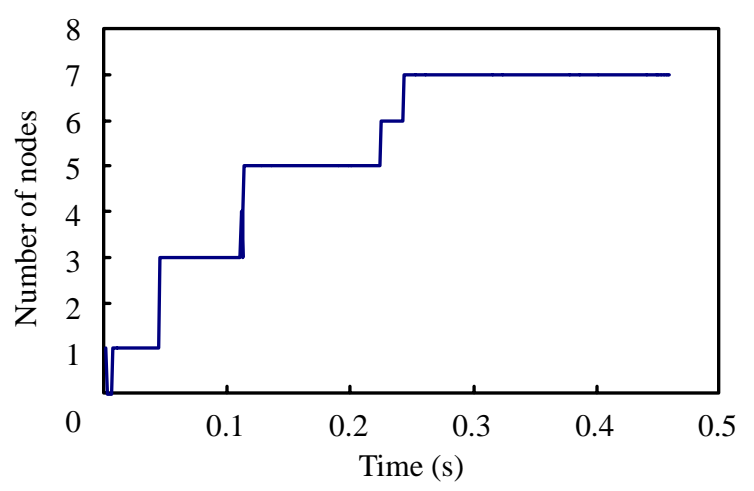

(a) Fifth ridge

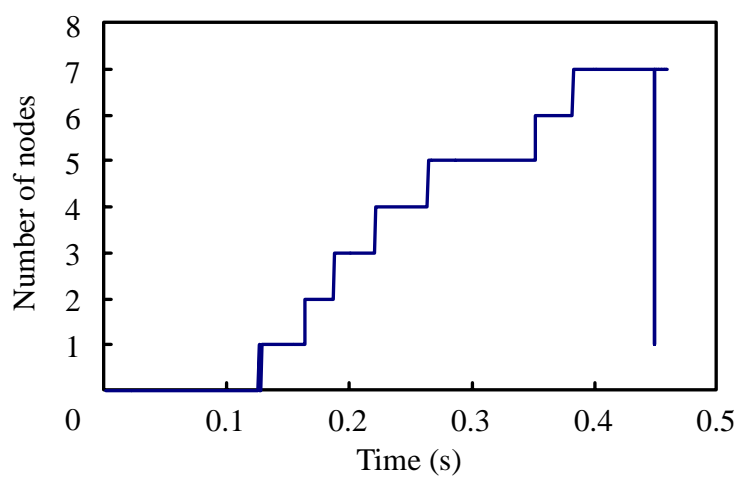

(b) Third ridge

Fig. 7 Number of nodes which sticks incipient slippage easily occurs from 0.4 to $0.5 \mathrm{~s}$ because a ratio $F_{t} / F_{n}$ is approaching the friction coefficient $\mu$. Slip area must gradually increase from the edge of the contact area at that time. The total time of the analysis was $0.46 \mathrm{~s}$ at which time entire slippage occurs. The kinetic friction coefficient was 1.0 and the static friction coefficient was 1.05.

The above-mentioned condition is called a "basic case". Analyses for some other cases were also conducted when friction coefficient, maximum value of grasping force and total time throughout the analysis were changed.

\subsection{Results of $\mathrm{FE}$ analysis}

First, an analysis for the basic case was conducted. Histories of the number of sticking nodal points in the third and fifth ridge are shown in Fig. 7. However, nodal point is a nodal point between each finite element. The number of sticking nodal points of both the third and fifth ridge was zero at first, and gradually increases to seven. This gradual increase of the stick area is due to the gradual increase of contact area caused by increased grasping force. The increment of the stick area is almost constant because it depends on the viscosity of the artificial finger skin material. The number of sticking nodal points of the

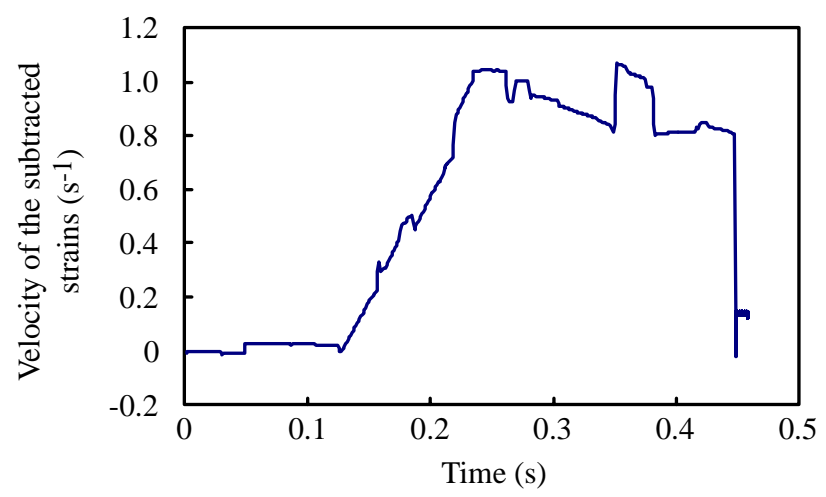

Fig. 8 Velocity of the subtracted strains inside third ridge for basic case

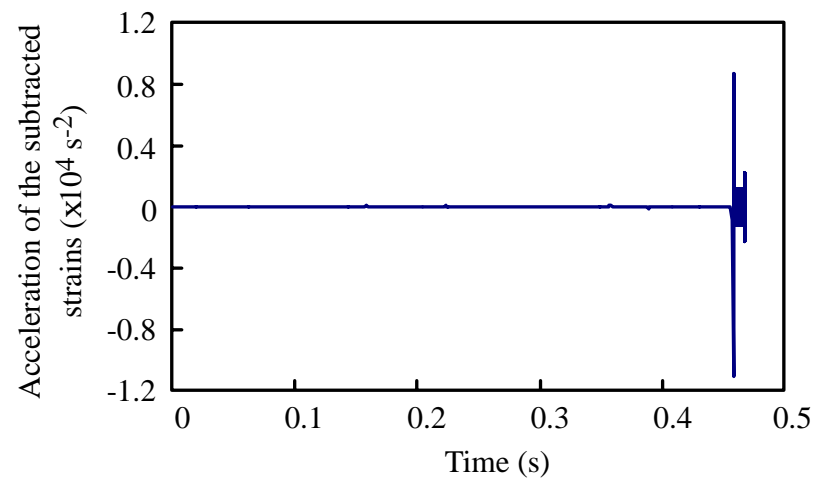

Fig. 9 Acceleration of the subtracted strains inside third ridge for basic case 
third ridge is suddenly decreased at the time $0.45 \mathrm{~s}$ as shown in Fig. 7 (b) because the entire slippage of the ridge occurs. However, that of the fifth ridge is not changed, i.e. the fifth ridge is not slipping. This means that only the ridges at the edge of the contact area slip as expected from the reasoning in section 2 . The predicted phenomena were seen in the results of the FE dynamic contact analysis.

The first and second time derivatives of the subtracted strains inside the third ridge, which we call the velocity and acceleration of subtracted strains, are shown in Fig. 8 and Fig. 9, respectively. The velocity of the third ridge increases gradually over time from $0.13 \mathrm{~s}$ to $0.23 \mathrm{~s}$. Then, it decreases when the incipient slippage occurs at time $0.45 \mathrm{~s}$, because the tangential deformation of the third ridge is released when the entire slippage occurs at the ridge. The acceleration of the third ridge responds with large amplitude at time $0.45 \mathrm{~s}$ as shown in Fig 9, because the velocity decreases at that time. The absolute value of the amplitude of the acceleration is larger than $4.0 \times 10^{3} \mathrm{~s}^{-2}$. On the other hand, the large response of the acceleration is not seen in the fifth ridge at that same time, because the entire slippage of the fifth ridge doesn't occur, even when the slippage of the third ridge occurs.

Analyses for the case when the applied force, the friction coefficient and the increment of applied force are changed were also conducted. The observed phenomena agree well with those of the basic case, even when the parameters are changed. The incipient slippage of the ridges near the edge of contact area occurs when the contact condition is changed from "stick" to "slip". Stepwise decreases of the velocity of subtracted strains were seen at the ridge near the edge of contact area when the entire slippage of the ridge occurs. The absolute value of the amplitude of the acceleration was always larger than $4.0 \times 10^{3} \mathrm{~s}^{-2}$ when the entire slippage of a certain ridge occurred. Large acceleration is not seen at other times.

Consequently, the velocity of subtracted strains inside the ridge near the edge of contact area greatly decreases when the contact condition changes from "stick" to "slip" due to the entire slippage of the ridge.

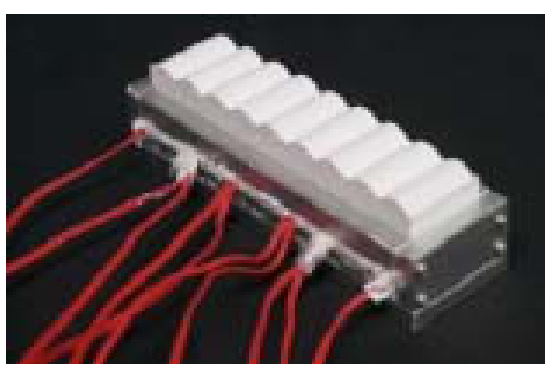

Fig. 10 Artificial finger skin
Therefore, the partial incipient slippage can be detected by the change in velocity and the absolute value of the acceleration of the ridges near the edge of the entire contact area as expected from section 2 .

\section{Experimental analysis}

\subsection{Equipment and experimental conditions}

The artificial elastic finger skin (Fig. 10) was produced to confirm the results of FE analyses. It consists of a silicone rubber whose material properties are the same as the FE model described in section 2.2. Strain gauges are embedded underneath each ridge at the location and direction shown in Fig. 3 . The measurement system of the experiment was as follows: The produced artificial finger skin was connected to a pedestal to fix it on the ground. A flat aluminum object was fixed on an $x-y$ stage. Then, external forces were applied to the artificial finger skin by controlling the position of the aluminum plate. The position of the $x-y$ stage was controlled by computer in order to apply the same external forces as those of the numerical analyses shown in Fig. 6 .

\subsection{Experimental results}

Figures 11 and 12 respectively show histories of

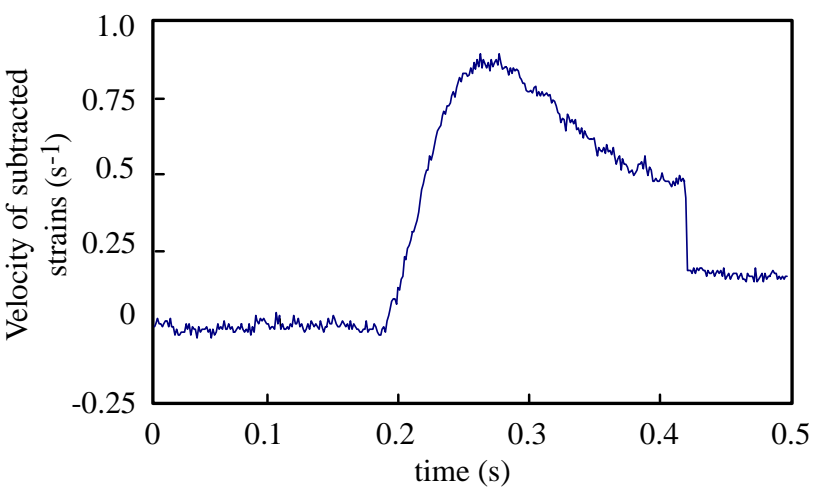

Fig. 11 Velocity of the subtracted strains inside third ridge in experiment

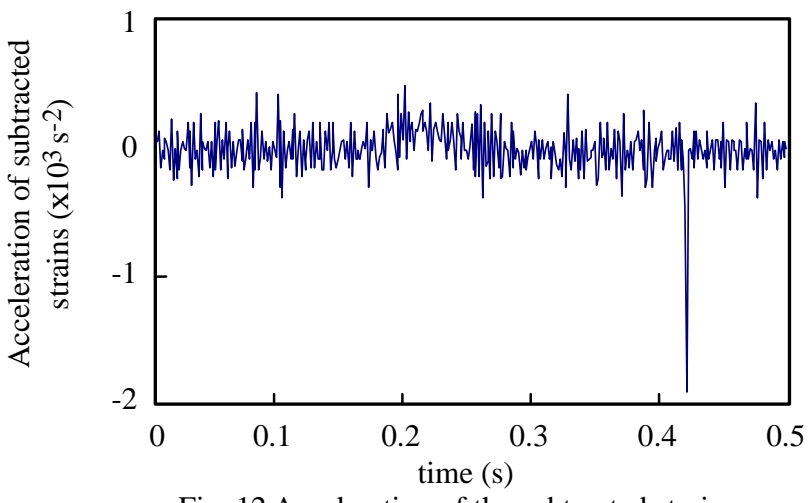

Fig. 12 Acceleration of the subtracted strains inside third ridge in experiment 


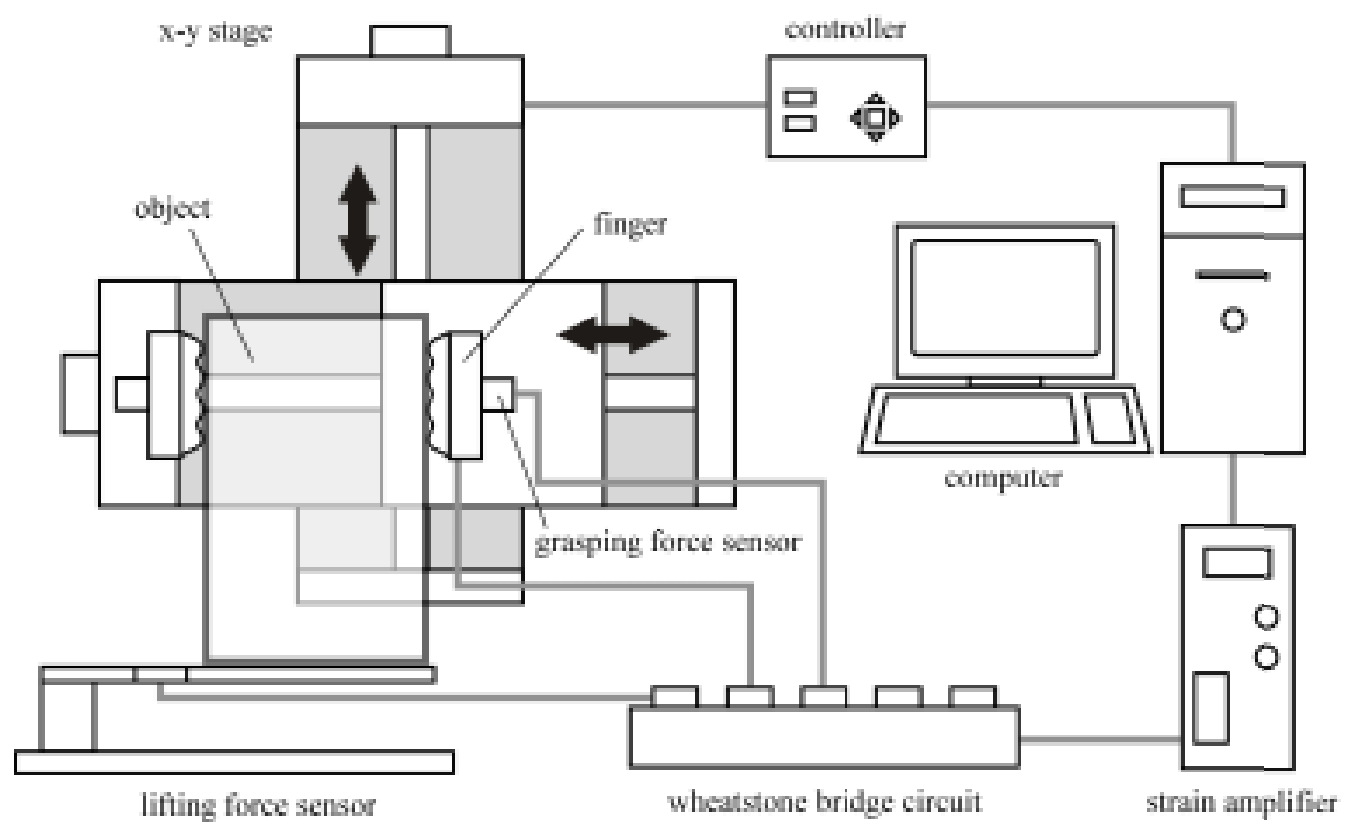

Fig.13 Equipment of experiment

the velocity and acceleration of subtracted strains inside the third ridge. These results are similar to those of the numerical analyses shown in Fig. 8 and Fig. 9. Particularly, when the entire slippage occurs at the third ridge, the velocity greatly decreases and the acceleration shows a negative impulse. Therefore, we can conclude that the results of the analyses are confirmed and the incipient slippage of the ridge near the edge of contact area can be detected by the change in the velocity and the acceleration. Since the acceleration due to the slippage of the ridge shows an impulse, PVDF films are an adequate sensor to detect this information in future study.

\section{Grasp force control}

\subsection{Equipment and procedure}

An experiment was conducted for controlling a grasp force. Figure 13 shows an equipment of experiment. Mass of grasped object is $0.91 \mathrm{~kg}$. Static frictional coefficient is 0.37 . Shape of the object is a rectangular parallelepiped. Grasping force is controlled by monitoring the stick/slip condition of each ridge. The control procedure is as follows: First, the grasping force $F_{n}$ is applied. Then, the $F_{n}$ and $F_{t}$ are increased. Increase ratio of $F_{n}$ is set to be larger than that of $F_{t}$ for preventing from slippage at the beginning of grasp. Then, the increase ratio of $F_{t}$ is increased gradually to be larger than that of $F_{n}$. If the slippage at the ridge is detected, increase ratio of $F_{t}$ is decreased so as to prevent the entire slippage. Then the above mentioned procedure is repeated. Objects whose weight and friction coefficient are unknown

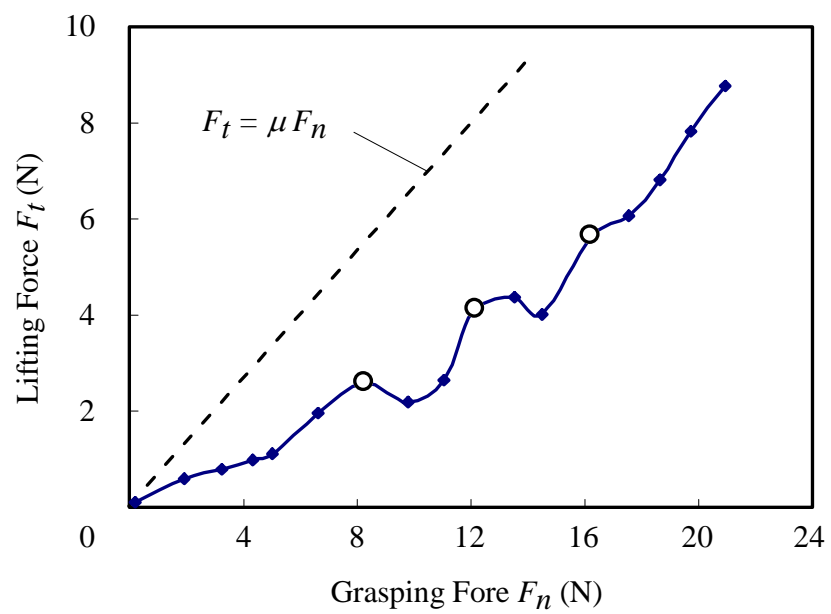

Fig.14 History of controlled external force

can be grasped and lifted by continuing this procedure.

\subsection{Results}

Figure 14 shows a history of the grasping force $F_{n}$ and lifting force $F_{t}$. White circles in Fig. 14 are points where the incipient slippage occurs at the edge of contact area. It can be concluded that the grasping force is controlled adequately and an object whose weight and friction coefficient are unknown is lifted without entire slippage. Unlike the previous finger, arbitrary objects can also be lifted by use of this 
control procedure at an arbitrary speed. If the weight of the object is changed while lifting the object, the finger can detect a partial slippage due to the change in the weight of the object.

The finger can grasp objects whose surface is almost flat. Objects whose surfaces have complex geometry have not been tested to be grasped. Objects which are softer than the finger have not been tested to be grasped, neither. The finger should be tested for grasping object which has complex geometry or is softer than the finger in the future study.

The method is considered to be the same as that of humans because humans seem to detect the partial insipient slippage wherever the contact surface exists. The proposed method for detecting the partial incipient slippage should be extended to develop a two-dimensional finger pad in the future study.

\section{Conclusion}

An elastic finger skin has been proposed for grasping an object whose weight and friction coefficient are unknown. It has a unique geometry imitating the configuration of ridges and FA I receptors of human fingers. First, the precise geometry of the artificial finger skin was designed using FE analysis. Then, it was confirmed by FE analysis and experiment that the incipient slippage of the ridges at the edge of the contact area can be detected by change in velocity and acceleration of the subtracted value of strains inside the ridges. Finally, the grasp force control is achieved. Arbitrary object is lifted successfully by use of incipient slippage near the edge of the contact area.

\section{Acknowledgement}

This study was supported in-part by a Grant-in-Aid for Scientific Research (\#12450168) from the Japan Society for the Promotion of Science, The Ministry of Education, Culture, Sports, Science and Technology.

\section{Reference}

[1] T. Maeno, K. Kobayashi and N. Yamazaki, "Relationship between the Structure of Human Finger Tissue and the Location of Tactile Receptors", Bulletin of JSME International Journal, Vol. 41, No. 1C, pp. 94-100, 1998.

[2] R. S. Johansson and G. Westling, "Roles of Glabrous Skin Receptors and Sensorimotor Memory in Automatic Control of Precision Grip When Lifting Rougher of More Slippery Objects", Exp. Brain Res., Vol. 56, pp. 550-564, 1984.

[3] R. S. Johansson and G. Westling, "Signals in Tactile Afferents from the Fingers Eliciting Adaptive Motor Responses During the Precision Grip”, Exp. Brain Res., Vol. 66, pp. 141-154,
1988.

[4] M. R. Tremblay and M. R. Cutkosky, "Estimation of Friction Using Incipient Slip Sensing During a Manipulation Task", Proc. IEEE Int. Conf. Robotics and Automation, Vol. 1, pp. 429-434, 1993.

[5] G. Canepa, R. Petrigliano, M. Campanella and D. De Rossi, "Detection of Incipient Object Slippage by Skin-Like Sensing and Neural Network Processing", IEEE. Trans. on Systems, Man, and Cybernetics -- part B:Cybernetics, Vol. 28, No. 3, pp. 348-356, 1998.

[6] T. Maeno, K. Kobayashi and N. Yamazaki, "Sensing Mechanism of the Partial Incipient Slip at the Surface of Cylindrical Fingers During the Precision Grip", Proc. ASME Summer Bioengineering Conf., pp. 117-118, 1997.

[7] T. Maeno, T. Kawai and K. Kobayashi, "Analysis and Design of a Tactile Sensor Detecting Strain Distribution inside ad Elastic Finger", Proc. IEEE/RSJ Int. Conf. Intelligent Robots and Systems, pp. 1658-1663, 1998.

[8] T. Maeno, S. Hiromitsu and T. Kawai, "Control of Grasping Force by Detecting Stick/Slip Distribution at the Curved Surface of an Elastic Finger", Proc. IEEE Int. Conf. Robotics and Automation, pp. 3896-3901, 2000.

[9] Y. Yamada, Y. Morita and Y. Umetani, "Slip phase isolating : impulsive signal generating vibrotactile sensor and its application to real-time object re-grip control", Robotica, Vol. 18, No. 1, pp.43-49, 2000. 